\title{
AVALIAÇÃO HIGIÊNICO-SANITÁRIA DA MANIPULAÇÃO DE ALIMENTOS, A NIVEL RESIDENCIAL, A PARTIR DA OCUPAÇÃO DO RESPONSÁVEL PELO PROCESSAMENTO
}

\section{SANITARY INSPECTIONS HANDLING OF FOOD, THE LEVEL HOME, FROM THE OCCUPATION OF PERSON HANDLING}

\author{
Mary Helen Ribeiro dos Santos ${ }^{1}$; Guataçara dos Santos Junior ${ }^{2}$; \\ Eliana Aparecida Fagundes Queiroz Bortolozo ${ }^{3}$ \\ ${ }^{1,2,3}$ Universidade Tecnológica Federal do Paraná - UTFPR - Ponta Grossa- Paraná - Brasil - \\ mary_hrs@hotmail.com; guatajr@uol.com.br; bortolozo@utfpr.edu.br;
}

\begin{abstract}
Resumo
O presente estudo, levando em consideração a ocupação dos responsáveis pela manipulação de alimentos no âmbito domiciliar, teve o objetivo avaliar as condições higiênico-sanitárias onde ocorre o processamento . Foram avaliados aspectos de higiene dos alimentos, desde a aquisição da matéria-prima, condições de processamento, até aspectos físicos das cozinhas visitadas. Os resultados foram tratados estatisticamente, aplicando-se a técnica de regressão logística binária. Para os cálculos, foi utilizado o software Minitab na versão 15. Com base nos dados, foi possível inferir que os manipuladores do grupo A apresentam a seguinte probabilidade: de ter os alimentos não perecíveis em armazenamento considerado adequado, em proporção cinco vezes maior que os manipuladores do grupo B; de ter os alimentos perecíveis em armazenamento adequado, em proporção quatro vezes maior que os manipuladores do grupo $B$; e de manter a cozinha em condições de higiene considerada adequada, em proporção cinco vezes maior que os manipuladores do grupo B. Chegando-se a conclusão de que a ocupação do responsável pelo processamento dos alimentos não interfere na sua qualidade final, pois em ítens considerados críticos durante o processamento (armazenamento e higiene), o grupo A obteve melhores resultados que o grupo $B$.
\end{abstract}

Palavras-chave: manipulação de alimentos, higiene dos alimentos, estatisticamente.

\section{Introdução}

A saúde é um direito de todo cidadão, tal como se encontra expresso na Declaração Universal dos Direitos Humanos, promulgada em 1948 pela Organização das Nações Unidas (ONU). Mas, para que haja saúde, é fundamental que os alimentos sejam produzidos em quantidade e com qualidade apropriada (GERMANO e GERMANO, 2001; OLIVEIRA et al., 2008). 
Pois saúde e alimento estão estritamente relacionados; os avanços tecnológicos e o aumento do consumo resultaram na mudança dos padrões sanitários de toda a cadeia, com vistas a evitar ou diminuir os riscos de toxinfecções alimentares, a partir da qualidade e segurança dos alimentos (UNGAR, 1992, citado por GERMANO et al., 2000).

As Doenças Veiculadas por Alimentos (DVA's) constituem um grande e crescente problema de saúde pública no mundo. A Organização Mundial da Saúde (OMS) estima que, anualmente, mais de um terço da população, incluindo países desenvolvidos, são acometidos por surtos de DVA's (BENEVIDES e LOVATTI, 2004; CARMO et al., 2006). A maioria das DVA's tem origem microbiológica, atribuindo-se este fato à manipulação e condições higiênicas inadequadas (FAÇANHA et al., 2003).

Dados do Centro de Vigilância Epidemiológica do Estado de São Paulo (CVE), obtidos no período de 2001 a 2005, revelam que foram notificados 405 surtos de DVA's, dos quais 93 (23\%) ocorreram em residências, evidenciando a necessidade de ações educativas sobre práticas higiênicas e seguras com alimentos, nas cozinhas domiciliares (MENNUCCI et al., 2006). Os procedimentos de preparação e de manuseio dos alimentos nas residências podem acarretar perigos microbianos, sendo importante a análise destes perigos, como dados para orientar as práticas normais dentro de áreas ou grupos étnicos particulares. O manuseio dos alimentos nas residências é assunto de responsabilidade dentro da família (ICMSF/IAMS, 1997, citado por OLIVEIRA et al, 2007).

No Brasil, no período de 2000 a 2002, ocorreram 348 surtos, sendo 42\% de origem domiciliar. Destes, 35,3\% foram veiculados por Salmonella sp, 10\% por Staphylococcus aureus, e a maionese foi responsável por 31,2\% dos surtos (OPAS/SIRVETA, 2004, citado por LEITE e WAISSMANN, 2006).

A Tabela 1 apresenta os principais espaços de ocorrência de surtos de doenças veiculadas por alimentos entre os anos de 1978-1999, no estado do Paraná.

Tabela 1 - Locais de ocorrência de surtos de Doenças Veiculadas por Alimentos, notificados no Estado do Paraná, entre 1978 - 1999.

\begin{tabular}{lcccc}
\hline \multicolumn{1}{c}{ Local de ocorrência } & Número de surtos & \% & Total acumulado & \% \\
\hline Domicílios & 928 & 52,1 & 928 & 52,1 \\
Refeitórios comerciais & 291 & 16,4 & 1219 & 68,4 \\
Refeitórios industriais & 166 & 9,3 & 1385 & 77,8 \\
Escolas & 114 & 6,4 & 1299 & 84,2 \\
Festas comunitárias & 73 & 4,1 & 1572 & 88,3 \\
Outros & 209 & 11,7 & - & - \\
Total & 1781 & 100 & 1781 & 100 \\
\hline
\end{tabular}

Dentre os fatores que mais contribuem para a ocorrência de surtos de doença de origem alimentar destaca-se a falta de cuidados no processamento dos alimentos. Destacam-se como 
condições propícias ao desenvolvimento de microrganismos aquelas provocadas pela manipulação inadequada, má utilização da temperatura durante o preparo e conservação dos alimentos, higiene pessoal dos manipuladores, contaminação cruzada, deficiência na higienização dos equipamentos e utensílios, cocção insuficiente, preparo dos alimentos com demasiada antecedência, entre vários outros fatores (SILVA JUNIOR, 1995). A higiene dos manipuladores, a armazenagem de alimentos, a higiene do ambiente de trabalho e as etapas de pré-preparo e preparo dos alimentos são itens imprescindíveis para uma alimentação de boa qualidade (TORRES et al., 2007).

Levando-se em consideração a ocupação dos responsáveis pela manipulação de alimentos no âmbito domiciliar, este estudo teve por objetivo avaliar as condições higiênico-sanitárias onde ocorre o processamento.

\section{Material e Métodos}

O estudo foi desenvolvido durante os meses de setembro a dezembro do ano de 2007, com base em estudo realizado "in loco", para avaliação das condições higiênico-sanitárias em 60 residências de famílias de crianças matriculadas em escolas municipais da cidade de Ponta GrossaPR.

Foram avaliados aspectos de higiene dos alimentos, desde a aquisição da matéria-prima, condições de processamento, até aspectos físicos das cozinhas visitadas.

A metodologia empregada constou de análise visual e preenchimento de "check-list", compreendendo itens que foram respondidos pelos responsáveis pela manipulação dos alimentos e incluíram dados referentes às características da moradia, às condições de armazenagem de produtos perecíveis e não perecíveis, às condições de higiene da cozinha, equipamentos e utensílios, higiene no processamento, conhecimentos sobre higiene do manipulador, entre outros. Para cada item foi fixado um peso (valor numérico), sendo o entrevistador treinado para que houvesse um padrão de avaliação.

O valor da pontuação para cada item do "check-list" foi assim padronizado:

- Satisfatório: quando a pontuação variou entre 70 e 100 pontos;

- Insatisfatório: quando a pontuação foi inferior a 70 pontos.

Para o "check list" foram utilizadas questões abertas, fechadas e/ou combinadas. A questão aberta deve ser utilizada quando se deseja diagnosticar determinado assunto, mas não se está familiarizado com a população a ser entrevistada e não se pode, portanto, antecipar possíveis respostas. Uma das vantagens das questões abertas é a possibilidade de o entrevistado responder com mais liberdade, não estando restrito a escolher uma ou outra alternativa (RICHARDSON, 1999). 
De acordo com Rodrigues et al. (2007), a utilização da análise de regressão logística binária, além de possibilitar a classificação de fenômenos ou indivíduos em categorias específicas, também pode estimar a probabilidade de que um determinado evento venha a se enquadrar nesta ou naquela categoria. Desta forma, os resultados obtidos para a variável em análise permitem interpretações que incluem probabilidade, e não somente classificação em categorias. Para os cálculos referentes à análise de associação entre a variável dependente e as independentes, assim como análise de razão de chances entre as observações, foi utilizado o software Minitab 15. As inferências para as análises pretendidas foram realizadas considerando nível de significância máximo de 0,05 , ou nível de confiança mínimo de 95\%. Outros detalhes sobre a regressão logística podem ser encontrados, por exemplo, em Devore (2006), Mingoti (2006), Pagano e Gauvreau (2008) e Motta (2006).

\section{Resultados e Discussão}

Dos 60 entrevistados responsáveis pela manipulação dos alimentos, 24 compunham o grupo dos que trabalhavam fora (Grupo A), e os 36 restantes não trabalhavam fora (Grupo B).

No item "Condições gerais da residência", o conceito "satisfatório" foi alcançado por 50\% dos manipuladores do grupo A e $32 \%$ do grupo B. Os fatores que influenciaram negativamente sobre o resultado das condições consideradas irregulares foram, entre outros, a falta de esgoto, ausência de geladeira e/ou freezer, piso sem revestimento, higiene inadequada. Vale ressaltar que a área física e as instalações são fatores que influenciam a higiene dos alimentos e possíveis riscos de contaminação dos mesmos (SILVA JUNIOR, 1995; MORAES, 1999, citado por DITZEL, 2006; MEDEIROS, 2001, citado por LEITE e WAISSMANN, 2006).

No item "Condição de armazenamento de produtos não perecíveis", o conceito "satisfatório" foi alcançado por $57 \%$ dos manipuladores do grupo A e $30 \%$ do grupo B. Os fatores que influenciaram negativamente nos resultados foram, entre outros, o armazenamento de produtos alimentícios em espaço comum com produtos de higiene e limpeza, falta de verificação do prazo de validade, área física de estocagem inadequada. Estes são pontos que interferem na qualidade dos alimentos, pois, quando expostos a condições ambientais inadequadas, normalmente sofrem a ação de fatores físicos e biológicos desse ambiente, o que pode propiciar a sua contaminação (CHESCA et al., 2001).

No item "Armazenamento de produtos perecíveis", o conceito "satisfatório" foi atingido por $42 \%$ dos manipuladores do grupo A e $15 \%$ do grupo B. Foram observadas situações, como falta de controle da validade dos produtos, armazenamento inadequado, procedimentos incorretos. Dados epidemiológicos demonstram que a conservação inadequada de alimentos perecíveis representa fator contribuinte para surtos de DVA's (CVE, 2007). O resultado deste estudo veio ao encontro de 
outros trabalhos que igualmente apontaram ineficiência do armazenamento de alimentos perecíveis, sobretudo de origem animal (SILVA JUNIOR, 1995; MEDEIROS, 2001, citado por LEITE e WAISSMANN, 2006; MURMANN et al., 2004).

No caso específico da "Conservação da carne", 15\% dos manipuladores do grupo A obtiveram conceito "satisfatório", enquanto que 7\% dos manipuladores do grupo B obtiveram esse conceito. $\mathrm{O}$ fator que mais influenciou negativamente foi o descongelamento, o qual vinha sendo geralmente realizado em temperatura ambiente, por tempo excessivamente prolongado. $\mathrm{O}$ descongelamento inadequado é um fator de risco para a qualidade do alimento. Relatos da literatura afirmam que temperaturas inadequadas de produtos perecíveis são uma das principais causas de contaminação (CHESCA et al., 2001; OLIVEIRA et al., 2003; SILVA et al., 2007)

No quesito "Conservação do leite e seus derivados", 45\% dos manipuladores do grupo A e $22 \%$ do grupo B, obtiveram conceito "satisfatório".

No caso dos "Hortifrutigranjeiros", o conceito "satisfatório" foi obtido por $52 \%$ dos manipuladores do grupo A e $32 \%$ do grupo B. O fator que mais influenciou negativamente a conceituação foi a higienização e sanitização insuficientes. Os relatos das entrevistas revelaram ser pouco observada a questão da sanitização, em virtude de que a maioria dos manipuladores não sabia preparar e nem utilizar a solução sanitizante. Higienização e sanitização de hortaliças podem reduzir os casos de contaminação, não apenas por bactérias e vírus, mas também por parasitas (SEMUS/PMV, 2006, citado por TAVARES, 2007).

No item "Condições de higiene da cozinha", 30\% dos manipuladores do grupo A obtiveram conceito satisfatório, o qual chegou a $7 \%$ entre os manipuladores do grupo B. As condições de higiene da área de manipulação de alimentos são de grande importância, sendo que pequenos cuidados podem evitar a contaminação dos produtos, principalmente nas cozinhas residenciais, onde são realizadas diversas atividades que oferecem risco de contaminação (CHIARINI e ANDRADE, 2004; LEITE e WAISSMANN, 2006; MENNUCCI et al., 2006).

Considerando a "Periodicidade de higienização de geladeira e freezer", foi atingido o conceito "satisfatório" por $25 \%$ dos manipuladores do grupo A e $10 \%$ do grupo B. Algumas inadequações foram observadas: a geladeira e/ou freezer somente eram descongelados quando a quantidade de gelo era muito grande; não se observava um critério na disposição correta dos alimentos nas prateleiras. O principal item que influenciou negativamente este resultado foi o processo de descongelamento, sendo que a maioria das entrevistadas desligava a geladeira à noite, para limpar na manhã seguinte, deixando os alimentos dentro da mesma, possibilitando risco de proliferação microbiana (SILVA et al., 2007).

Tratando-se da "Área interna", 43\% dos manipuladores do grupo A e 27\% do grupo B obtiveram conceito "satisfatório". Os principais pontos negativos observados antes da capacitação 
foram a permissão de animais domésticos na cozinha e a higienização inadequada. Trata-se de fatores relevantes, uma vez que a higienização incorreta da cozinha pode ensejar a contaminação de alimentos, utensílios e equipamentos, sendo que os animais domésticos podem veicular várias doenças (SILVA JUNIOR, 1995; CHIARINI e ANDRADE, 2004).

No "Manejo de resíduos", 37\% dos manipuladores do grupo A obtiveram conceito "satisfatório", enquanto que $18 \%$ dos manipuladores do grupo B obtiveram esse conceito. Foram consideradas como inadequações: coletores sem tampa dispostos na cozinha e/ou em cima da pia; acúmulo de resíduos e descarte pouco frequente. Sabe-se que os resíduos representam fator importante de contaminação e que, mesmo em empresas processadoras de alimentos, muitas vezes não se observam cuidados quanto à qualidade do material de reserva dos resíduos, sua remoção frequente, e mesmo seu destino (HAZELWOOD e MCLEAN, 1994). Conforme foi observado em muitas residências, o resíduo sólido (lixo) não vinha recebendo a necessária atenção, podendo representar foco de infestação de pragas e veículo de contaminação dos alimentos (SERVSAFE, 2000; FAÇANHA et al., 2003).

$\mathrm{Na}$ conservação de "Higiene da caixa d'água", 23\% dos manipuladores do grupo A obtiveram conceito "satisfatório", o mesmo para 8\% do grupo B. Vários outros estudos demonstraram resistência a este cuidado, mesmo em estabelecimentos comerciais. Em algumas regiões do país, até mesmo a água do sistema de abastecimento público pode apresentar-se contaminada, pelo descuido com a caixa d'água (MICHELINA et al., 2006). Assim sendo, todos os cuidados higiênicos podem ser perdidos, se a água utilizada na cozinha for de má qualidade (SILVA JUNIOR, 1995).

No caso das "Condições de higiene dos utensílios", o conceito "satisfatório" foi alcançado por $45 \%$ dos manipuladores do grupo A e $25 \%$ do grupo B. Algumas situações foram consideradas inadequadas, como por exemplo: a utilização de utensílios de madeira, higienização insuficiente e utilização de um mesmo pano de prato para várias operações durante o processamento dos alimentos. Constatou-se, porém, que, após receber orientação, o responsável pela higienização destes materiais mostrou disposição para melhorar sua conduta. Vale lembrar que equipamentos e utensílios em estado inadequado de higiene veiculam diversos microrganismos. Desire e Eduardo (2001) detectaram em pesquisa a contaminação de equipamentos por coliformes totais e fecais. De acordo com a literatura, os panos de prato podem se tornar veículos para contaminação cruzada (OLIVEIRA et al., 2007).

Considerando as "Condições de preparação dos alimentos", 42\% dos manipuladores do grupo A obtiveram conceito satisfatório, enquanto que $25 \%$ dos manipuladores do grupo B obtiveram esse conceito. Vários dados da literatura comprovam que a postura do manipulador é fundamental para se reduzirem surtos de DVA's. Falhas detectadas neste estudo, como manipulação 
inadequada, preparo dos alimentos com demasiada antecedência, manutenção dos alimentos a temperatura ambiente e reprocessamento de alimentos, foram também observadas em estudos de outros autores (GÓES et al., 2001; OLIVEIRA et al., 2002, citados por OLIVEIRA et al., 2003; VALEJO et al., 2003).

Quanto ao item "Higiene pessoal", o conceito "satisfatório" foi atingido por 50\% dos manipuladores do grupo A e $40 \%$ do grupo B. Foram observadas algumas inadequações que influenciaram negativamente a avaliação, tais como: a realização de outras tarefas ao mesmo tempo em que se manipula o alimento; os cabelos presos, mas desprotegidos e portando adereços. Hábitos de higiene, como lavar as mãos, acabam passando despercebidos, quando o responsável pelo preparo do alimento na residência executa concomitantemente outras atividades, o que pode gerar contaminação cruzada (PANETTA, 1998; HAZELWOOD e MCLEAN, 1994; GUIMARAES et al., 2007).

No item "Nível de conhecimento sobre higiene", o conceito "satisfatório" foi de $22 \%$ entre os manipuladores do grupo A e $12 \%$ entre os manipuladores do grupo B.

Complementando a análise dos dados, e tendo-se em vista a forma de tratamento dos mesmos, foram consideradas dependentes duas das variáveis trabalhadas: o responsável pela manipulação dos alimentos trabalhar fora (Grupo A), e o responsável pela manipulação dos alimentos não trabalhar fora (Grupo B). Tornou-se importante avaliar uma possível associação entre as variáveis consideradas dependentes e os resultados obtidos para as outras variáveis. Utilizando-se o software Minitab versão 15, foi então realizada a análise de regressão logística binária, pelo fato de se estar trabalhando com variáveis dependentes consideradas dicotômicas, isto é, de natureza binária, e que são, além disso, variáveis de natureza qualitativa.

A análise pelo software Minitab 15 demonstrou que apenas as variáveis “Armazenamento de produtos perecíveis", “Armazenamento de produtos não perecíveis" e "Higiene da cozinha" parecem ser dependentes das variáveis representadas pelo fato do responsável pela manipulação dos alimentos trabalhar fora (Grupo A), ou não (Grupo B). Parece haver associação significativa, com 97\% de confiança, entre o fator "trabalhar fora ou não" e o item armazenamento dos produtos não perecíveis. O mesmo se pode afirmar, mas com 99,1\% de confiança, sobre associação entre "trabalhar fora ou não", com a variável higiene da cozinha. Também há fortes indícios de haver associação entre "trabalhar fora ou não" e o quesito produtos perecíveis, afirmação feita com 98,6\% de confiança.

Com base no exposto, pode-se inferir que os manipuladores do grupo A apresentam: probabilidade de ter os alimentos não perecíveis em armazenamento considerado adequado, numa proporção cinco vezes maior do que os manipuladores do grupo B; de ter os alimentos perecíveis 
em armazenamento adequado, em nível quatro vezes maior que os do grupo B; e de ter a higiene da cozinha considerada adequada, num parâmetro cinco vezes maior do que o grupo B.

\title{
4. Conclusão
}

Pode-se inferir que os manipuladores do grupo A apresentam probabilidade cinco vezes maior, de ter os alimentos não perecíveis em armazenamento considerado adequado, se comparados aos manipuladores do grupo B (associação com $\mathrm{p}=0,03$ ); o grupo A pode ter os alimentos perecíveis em armazenamento adequado, quatro vezes maior que o grupo B (associação com p=0,014) e também pode ter a higiene da cozinha considerada adequada, cinco vezes maior que o grupo B (associação com p=0,009).

Assim, pode-se concluir que a ocupação do responsável pelo processamento dos alimentos não interfere na sua qualidade final, pois em ítens considerados críticos durante o processamento (armazenamento e higiene), o grupo A (trabalham fora) obteve melhores resultados que o grupo B (não trabalham fora).

\begin{abstract}
This study, taking into account the occupation of those responsible for food handling in the home, was aimed to evaluate the sanitary conditions where processing occurs. We assessed aspects of food hygiene, since the acquisition of raw material, processing conditions, by physical aspects of kitchens visited. The results were statistically treated by applying the technique of binary logistic regression. For calculations, we used the software Minitab version 15. Based on the data, we suggest that the handlers of group $A$ had the following probability: to take nonperishable food in storage as adequate, in proportion to five times greater than the manipulators of group $B$ have the proper storage of perishable foods as a proportion four times greater than the manipulators of the group $B$ and keep the kitchen in a hygienic considered appropriate to rate five times higher than the manipulators of the group B. He came to the conclusion that the occupation of the person responsible for processing the food does not interfere in their final quality as on items considered critical during processing (storage and hygiene), group A achieved better results than the B group.

Key-words: food handling, food hygiene, statistically.
\end{abstract}

\section{Referências}

BENEVIDES, C. M. J.; LOVATTI, R. C. C. Segurança Alimentar em estabelecimentos processadores de alimentos. Revista Higiene alimentar, v. 18, n. 125, p. 24-28, 2004.

CARMO, G. M. I.; DIMECH, C. P. N.; ALVES, R. M. S. Vigilância dos Surtos de doenças transmitidas por alimentos, Brasil, 1999-2006. Revista Higiene Alimentar, v. 21, n. 150, p. 353-354, 2006.

CHESCA, A. C.; PEIXOTO, C. P.; COSTA, D. G.; NASCIMENTO, H. N.; PINTO, I. R. A.; GUIMARÃES, J. L. P.; TARQUINIO, L. B.; OKURAI, M. H. Levantamento das temperaturas de armazenamento de carnes, em açougues e supermercados de Uberaba, MG. Revista Higiene alimentar, v. 15, n. 84, p. 51-55, 2001.

CHIARINI, E.; ANDRADE, C. S. Levantamento de procedimentos higiênicos adotados em cozinhas residenciais. Revista Higiene Alimentar, v. 18, n. 121, p. 34-38, 2004. 
CVE/SES-SP - Centro de vigilância Epidemiológica/Secretaria de Estado da Saúde de São Paulo. Manual das Doenças Transmitidas por Alimentos. Disponível em <www.cve.saude.sp.gov.br/htm/hidrica/shiguella.htm> acesso em 22-out-2007.

DESIRE, S.; EDUARDO C. T. Análise de perigos e pontos críticos de controle em uma unidade de alimentação e nutrição. Revista Higiene Alimentar, v. 15, n. 85, p. 41-49, 2001.

DEVORE, J. L. Probabilidade e estatística: para engenharia e ciências. São Paulo: Pioneira Thomson Learning, 2006.

FAÇANHA, S. H. F.; MONTE, A. L. S.; FERREIRA, N. D. L.; ALVES, T. M.; DIAS, G. M.; RIDRIGUEÊS, J. M. P.; PAULO, A. P. F. Treinamento para manipuladores de alimentos, em escolas da rede municipal de ensino, da sede e distritos do município de Meruoca, Ceará: Relato de experiência. Revista Higiene Alimentar, v. 17, n. 106, p. 30-34, 2003.

GERMANO, P. M. L. GERMANO, M. I.S. Características Fundamentais dos Alimentos. São Paulo: Livraria Varela, 2001.

GÓES, J. A. W.; FORTUNATO, D. M. N.; VELOSO, I. S.; SANTOS, J. M. Capacitação dos manipuladores de alimentos e a qualidade da alimentação servida. Revista Higiene Alimentar, v. 15, n. 82, p. 20-23, 2001.

HAZELWOOD, D.; MCLEAN, A. C. Manual de Higiene para manipuladores de alimentos. 1 ed., São Paulo: Livraria Varela, 1994.

ICMSF/IAMS. Comissão Internacional para Especificações Microbiológicas dos alimentos da União Internacional das Sociedades de Microbiologia. São Paulo: Livraria Varela, 1997. In: OLIVEIRA, L. C. J.; FARIA, K. N.; NEGREIROS, A. B.; TÓRTORA, J. C. O. Qualidade higiênico-sanitária de tábuas de corte, panos de prato e esponjas, em cozinhas residenciais. Revista Higiene Alimentar, v. 21, n. 156, p. 90-96, 2007.

LEITE, L. H. M.; WAISSMANN, W. Surtos de toxinfecções alimentares de origem domiciliar no Brasil, de 2000-2002. Revista Higiene Alimentar, v. 20, n. 147, p. 56-59, 2006.

MEDEIROS, L.C.; KENDALL, P.; HILLERS, V.; CHEN, G.; DIMASCOLA, S. Identification and classification of consumer food handling behaviors for food safety education. J. Am diet Assoc. v, 101, p. 1326-1339, 2001.

MENNUCCI, T. A.; SOUZA, T. A. M.; CHAABAN, H. M. A. Prevenção de doenças transmitidas por alimentos em cozinhas residenciais: uma abordagem educativa da Vigilância Sanitária de Diadema, Revista Higiene Alimentar, v. 21, n. 150, p. 372-373, 2006.

MICHELINA, A. F.; BRONHAROA, T. M.; DARÉB, F.; PONSANOC; E. H. G. Qualidade microbiológica de águas de sistemas de abastecimento público da região de Araçatuba, SP. Revista Higiene Alimentar, v. 20, n. 147, p. 90-95, 2006.

MINGOTI, S. A. Análise de dados de métodos de estatística multivariada: uma abordagem aplicada. Belo Horizonte. Editora UFMG, 2005.

MORAES, I. R. CVS/SES-SP. Manual de Doenças Transmitidas por Alimentos e Água, jun/99. In: DITZEL, E. A. $O$ perfil do manipulador de alimentos em unidades de alimentos e nutrição. Universidade Tecnológica Federal do Paraná, Campus Ponta Grossa, 2006.

MOTTA, V. T. Bioestatística. $2^{\circ}$ Ed. Caxias do Sul - RS: Educs, 2006.

MURMANN, L.; DILKIN, P.; KOWALSKI, C. H.; ALMEIDA, C. A.; MALLMANN, C. A. Temperaturas de conservadores a frio em estabelecimentos que comercializam alimentos, na cidade de Santa Maria/RS. Revista Higiene Alimentar, v. 18, n. 124, p. 30-34, 2004.

OlIVEIRA, A. M.; GONÇALVES, M. O.; SHINOHARA, N. K. S.; STAMFORD, T. L. M. Manipuladores de alimento: um fator de risco. Revista Higiene Alimentar, v. 17, n 114/115, p. 12-18, 2003. 
OLIVEIRA, L. C. J.; FARIA, K. N.; NEGREIROS, A. B.; TÓRTORA, J. C. O. Qualidade higiênico-sanitária de tábuas de corte, panos de prato e esponjas, em cozinhas residenciais. Revista Higiene Alimentar, v. 21, n. 156, p. 90-96, 2007.

OLIVEIRA, M. N.; BRASIL, A. L. D.; TADDEI, J. A. A. C. Avaliação das condições higiênico-sanitárias das cozinhas de creches públicas e filantrópicas. Revista de Saúde Coletiva, v. 13, n. 3, mai/jun 2008.

OPAS/SIRVETA - Oraganizacion Panamericana de la Salud. Sistema Regional de información para la vigilância de lãs enfermidades transmitidas por alimentos. Disponível em <www.panalimentos.org/sirveta> acesso em 16-out2004. In: LEITE, L. H. M.; WAISSMANN, W. Surtos de toxinfecções alimentares de origem domiciliar no Brasil de 2000-2002. Revista Higiene Alimentar, v. 20, n. 147, p. 56-59, 2006.

PAGANO, M.; GAUVREAU, K. Princípios de bioestatística. São Paulo: Cengage Learning, 2008.

PANETTA, J. C. O manipulador: fator de segurança e qualidade dos alimentos. Revista Higiene Alimentar, v. 12, n. 57, p. 08-10, 1998.

RICHARDSON, J. Roberto. Pesquisa Social: métodos e técnicas. São Paulo: Atlas, 1999.

RODRIGUES, A.; CORRAR, L. J.; PAULO, E.; DIAS FILHO, J. M. Análise multivariada: para os cursos de administração, ciências contábeis e economia. FIPECAFI - Fundação Instituto de Pesquisas Contábeis, Atuariais e Financeiras. São Paulo: Atlas, 2007.

SEMUS/PMV - Secretaria Municipal de Saúde/Prefeitura Municipal de Vitória-ES. As Boas Práticas na Manipulação de Alimentos: Artesãos da Culinária. Fev. 2006. Disponível em <www.vitoria.es.gov.br/secretarias/saude/cartilha_boas_praticas_artesao_alimentos.pdf> acesso em: 15-mar-2007. In:

TAVARES, A. C. Alimentos Seguros: condições higiênico-sanitárias na manipulação de alimentos em residências. Universidade Tecnológica Federal do Paraná, Campus Ponta Grossa, 2006.

SERVSAFE. Princípios básicos de segurança alimentar / Instituto de Hospitalidade. Rio de Janeiro: Instituto de Hospitalidade, 2000.

SESA/ISEP/CSA - Secretaria do Estado do Paraná/Instituto de Saúde do Paraná/Centro de Saúde Ambiental, 2007.

Silva, A. C. M.; ANDRADE L. P.; GUIMARÃES, K. A. S. Avaliação do binômio tempo x temperatura de preparações proteicas, durante processo produtivo numa unidade de alimentação e nutrição. Revista Higiene Alimentar, v. 21, n. 157, p. 70-75, 2007.

SILVA JUNIOR, E. A. Manual de controle higiênico-sanitário em alimentos. 4 ed. São Paulo: Livraria Varela, 1995.

TORRES, S. A. M.; SILVA, V. A.; COELHO, A. Í. M.; MIRANDA, A. S. Análise das condições higiênico-sanitárias durante o preparo da alimentação em cantina escolar. Revista Higiene Alimentar, v. 21, n. 153, p. 14-18, 2007.

UNGAR, M. L.; GERMANO, M. I. S.; GERMANO, P. M. L. Riscos e consequências da manipulação de alimentos para a saúde pública. Revista Higiene Alimentar, v. 6, n. 21, p. 14-17, 1992.

VALEJO, F. A. M.; ANDRÉS, C. R.; MANTOVAN, F. B.; RISTER, PEIXOTO, G. ; SANTOS, G. D.; ANDRADE, F. F. Vigilância Sanitária: avaliação e controle da qualidade dos alimentos. Revista Higiene Alimentar, v. 17, n. 106, p. 16-20, 2003.

Submetido em 27 out. 2010; Revisão submetida pelos autores em 21 mai. 2011; Aceito para publicação em 30 jun.2011. 